\title{
Observability of $s$-channel heavy charged Higgs at LHC using top tagging technique
}

\author{
Majid Hashemi and Gholamhossein Haghighat \\ Physics Department, College of Sciences, Shiraz University, \\ Shiraz 71454, Iran \\ E-mail: hashemi_mj@shirazu.ac.ir, hosseinhaqiqat@gmail.com
}

\begin{abstract}
In this paper, the question of observability of a heavy charged Higgs in the mass range $400 \mathrm{GeV}<m_{H^{ \pm}}<1000 \mathrm{GeV}$, is addressed. The production process is set to $p p \rightarrow H^{ \pm} \rightarrow t \bar{b}$ at $14 \mathrm{TeV}$ LHC. The analysis benefits from top tagging technique which is based on finding a fat jet as a result of the boosted top quark decay in signal events. A detailed hadron level analysis is performed and selection efficiencies are presented with different charged Higgs mass hypotheses. Finally running toy experiments and using pseudo-data, a fit over signal plus background distributions is performed to assess possibility of reconstructing the charged Higgs peak and its invariant mass measurement. It is shown that the charged Higgs mass can well be reconstructed in the mass range $500 \mathrm{GeV}$ to $1 \mathrm{TeV}$, with a signal significance which depends on $\tan \beta$. Eventually $5 \sigma$ discovery and 95\% C.L. exclusion contours are also provided.
\end{abstract}

KeYwords: Higgs Physics, Supersymmetric Standard Model

ArXIV EPRINT: 1511.00874 


\section{Contents}

1 Introduction 1

2 Single top events as sources of charged Higgs 2

3 Jet substructure and top tagging technique 3

4 Signal and background events and their cross sections $\quad 6$

$\begin{array}{lll}5 & \text { Event generation and analysis } & 7\end{array}$

$\begin{array}{lll}6 & \text { Signal significance and phase space contours } & 17\end{array}$

$\begin{array}{llr}7 & \text { Conclusions } & 18\end{array}$

\section{Introduction}

The charged Higgs boson is one of the particles whose observation would reveal the existence of models beyond the Standard Model of Particle Physics. Within the framework of Minimal Supersymmetric Standard Model (MSSM), the Large Hadron Collider (LHC) is currently performing search for this particle in the parameter space $\left(m_{H^{ \pm}}, \tan \beta\right)$ with no evidence still found. Here, $\tan \beta$ is the ratio of vacuum expectation values of the two Higgs doublets used to make the MSSM [1].

The theoretical and phenomenological studies which paved the way to experimental searches include studies of properties of the charged Higgs [2], observability at Tevatron [3], $\tau$ polarization studies [4-8], study of the $g b$ fusion as a source of charged Higgs production [9], the $t b$ decay channel study [10], heavy charged Higgs study at LHC [11], charged Higgs three body decay [12], pair production in gluon-gluon collisions [13], triple $b$-tagging as a tool for charged Higgs detection [14], charged Higgs production in $b g$ fusion $[15,16]$ and the associated production of top and charged Higgs [17]. These studies, all together, showed that the promising decay channel for a heavy charged Higgs is $t \bar{b}$ and $\tau \nu$, while for a light charged Higgs (lighter than the top quark), decay to $\tau \nu$ plays the main role. Of course when a $\tau$ lepton is produced in the event, use of the $\tau$ polarization makes it easy to distinguish between the charged Higgs boson signal and SM background events.

The more data LHC collects, the higher charged Higgs masses are accessible for analysis. This is due to the fact that all production processes are decreasing functions of the charged Higgs mass. Therefore charged Higgs masses near the TeV scale may need a large amount of data to observe and assuming its existence, the heavier the charged Higgs, the more challenging its observation. This is the reason of attempt, in this paper and few upcoming ones, for analysis of charged Higgs near the TeV scale. The result of this analysis is 
expected to help LHC experiments in their progress to this region of parameter space. LHC data will soon provide opportunity to explore heavy charged Higgs boson and the analysis to be presented in this paper and similar ones would reveal their importance when working in that area of parameter space. It may be notorious that accroding to flavour Physics studies and global fits to experimental data, a charged Higgs with $m_{H^{ \pm}} \simeq 600 \mathrm{GeV}$ at low $\tan \beta$ values fits well with EW and flavour Physics observations and the overall deviation of theory (2HDM type II) from experiment in terms of a global $\chi^{2}$ is less than that of SM [18].

In order to establish where we are and where we are going, a presentation of current results of collider searches for the charged Higgs follows. Currently there are several main limits on the mass of the charged Higgs. There is a $\tan \beta$ independent low mass limit of $m_{H^{ \pm}}>78.6 \mathrm{GeV}$ from direct searches at LEP [19-21] while their indirect searches set a limit of $m_{H^{ \pm}}>125 \mathrm{GeV}$ [22]. The latter is used in this analysis as it covers the former. The Tevatron searches by D0 [23-26] and CDF Collaborations [27-29] exclude high tan $\beta$ values. The scope of such analyses is the light charged Higgs which is now confirmed and extended by LHC searches.

LHC experiments (CMS and ATLAS) started light and heavy charged Higgs analyses based on Monte Carlo simulations in separated works. Light charged Higgs possibility of observation was studied by CMS [30] and ATLAS [31] using the top pair production process with a top quark decaying to charged Higgs. Such an assumption is, however, almost excluded according to LHC real data analyses performed by CMS [32, 33] and ATLAS [34, 35]. Their conclusion is that a charged Higgs with $m_{H^{ \pm}}<160 \mathrm{GeV}$ is excluded at $95 \%$ C.L. for almost all $\tan \beta$ values.

Heavy charged Higgs Monte Carlo analyses at CMS [36, 37] and ATLAS [38, 39] have been based on associated production of the charged Higgs and a top quark, i.e., $g \bar{b} \rightarrow t H^{-}$, with special care of both $H^{ \pm} \rightarrow \tau \nu$ and $H^{ \pm} \rightarrow t \bar{b}$ decay channels. The ongoing LHC direct searches also rely on the same production process and decay in the search for heavy charged Higgs [40, 41]. There has been also indirect searches for the charged Higgs through the observation of deviation from what is expected from SM $t \bar{t}$ events [42]. However result of the analysis presented in [42] is superceded by direct search results [40, 41] which exclude heavy charged Higgs masses in the range $m_{H^{ \pm}}=180-230 \mathrm{GeV}$ at high $\tan \beta$ values.

\section{Single top events as sources of charged Higgs}

In a couple of previous works, single top events were proved to be viable sources of light and heavy charged Higgs in $t$-channel and $s$-channel single top production respectively [43, 44]. The heavy charged Higgs can be produced as a resonance in $s$-channel single top production, i.e., $p p \rightarrow H^{ \pm} \rightarrow t \bar{b}$ with the possibility of reconstructing the charged Higgs invariant (transverse) mass in hadronic (leptonic) final state. The analysis presented in [44] relies on the leptonic final state which is related to the leptonic decay of the $\mathrm{W}$ boson in the top quark decay. It has led to promising results relevant to $200 \mathrm{GeV}<m_{H^{ \pm}}<400 \mathrm{GeV}$. However, the analysis fails for higher charged Higgs masses at low luminosity of LHC due to the small cross section of production process. Including off-diagonal contribution of incoming quark pair has also been shown to improve the results sizably [45]. However, one 
may need to use technical tools for heavier charged Higgs bosons in order to improve the signal significance and extend the analysis to TeV scale charged Higgs.

In what follows, we try to describe the top tagging technique and its application to selection of signal events which contain a boosted top quark. The top quark may get a large boost in decay of a heavy particle from beyond Standard Model, like MSSM charged Higgs, which decays to $t \bar{b}$ if its mass is above the kinematic threshold. As an example, a top quark from a charged Higgs decay with $m_{H^{ \pm}}=700 \mathrm{GeV}$ gets a much larger Lorentz boost compared to what it acquires in SM events like $t \bar{t}$. Identifying such top quarks using top tagging technique which is based on sub-jet identification in the top quark hadronic decay may help a lot in discrimination of BSM events and their characteristic particles.

\section{Jet substructure and top tagging technique}

Due to its high center of mass energy, LHC produces highly boosted particles in its detectors frames. When such energetic particles decay, their decay products tend to be collinear due to the Lorentz boost they receive at the laboratory rest frame. In case of top quark which decays before it can hadronize [46], this situation results in collinear jets in the fully hadronic decay $t \rightarrow W^{+} b \rightarrow j j b$, i.e., two light jets from the $\mathrm{W}$ boson decay and a $b$-jet directly from the top quark decay. Identification of such jets by present jet reconstruction algorithms is a challenging task due to not being well separated from each other. A cone of $\Delta R=\sqrt{(\Delta \eta)^{2}+(\Delta \phi)^{2}}=0.4$ for jet reconstruction can not distinguish well each one of the three jets from the top quark decay. In this case, a top quark decay appears as a "fat" jet spreaded in a large cone of $\Delta R \simeq 1.5$. Therefore a jet algorithm should start from a large cone of that size, identify the fat jet, and preferably check the invariant mass of "sub-jet" pairs. By definition, a sub-jet is a jet inside the large cone of the highly boosted particle, in this case the top quark, associated with either the $b$-jet or the two light jets from the $\mathrm{W}$ hadronic decay.

From the Physics point of view, few percent of top quarks from Standard Model (SM) events may acquire enough boost to appear as a fat jet [47]. However, a massive $s$-channel resonance from beyond SM, may produce highly boosted top quark in its decay. An example of such a case, relevant to this work, is a massive $s$-channel charged Higgs production, $p p \rightarrow H^{ \pm} \rightarrow t \bar{b}$ whose final state particles are similar to the $s$-channel SM single top but with a large difference in kinematics. A charged Higgs with a mass of a reasonable fraction of $\mathrm{TeV}$, produces a top quark which is well distinguishable from the $s$-channel SM single top. Such a jet identification and reconstruction receives a negligible fake rate from QCD multi-jet events and electroweak gauge boson production processes due to the high kinematic threshold used for BSM signal events.

In recent years, special care has been devoted to the so called "top tagging" technique. With the more data LHC provides to its experiments, CMS and ATLAS, searches for heavier BSM particles becomes feasible. In some cases, searching for a heavy BSM particle requires not only more data but also special care in experimental analysis due to the high particle multiplicity and the associated combinatorial background. The high particle multiplicity may be the result of heavy electroweak gauge boson decays as well as top quark 
decays in their hadronic final state. The case of heavy charged Higgs, stated previously, is an example of such a situation. The top tagging algorithm has been designed to identify top quarks in such highly boosted regimes. It tries to deal with not only the high particle multiplicity in events, but also the collinearity of final state jets from the top quark decay. One of the early reports on introducing and applying boosted jet tagging technique is the one known as BDRS tagger [48]. It was originally designed for improving searches for associated Higgs production in $t \bar{t} H, W H$ and $Z H$ for the low mass Higgs when the Higgs boson decays to a $b \bar{b}$ pair. It was a successful application of tagging technique in which a large cone for the jet reconstruction was used to identify a fat jet and decompose it to two $b$-jets. This technique was shown to work better than the standard reconstruction techniques, due to moving the analysis to a highly boosted regime. Although only about $5 \%$ of events are produced with a reasonable boost (Higgs boson $p_{T}>200 \mathrm{GeV}$ ), several advantages led to a more promising result in that analysis: the larger $V H$ system mass resulted in more central events with decay products having higher transverse momenta to be tagged. SM background events were less likely to produce a high- $p_{T} b \bar{b}$ system consistent with the Higgs boson mass. Working in the highly boosted regime provided the opportunity to make the $Z H$ process with $Z \rightarrow \nu \bar{\nu}$ visible because of the large missing transverse energy.

In the current analysis, the same physics reasons motivate using a top tagger. A standard search may be dominated by the large QCD events due to their large cross section. Applying a high $p_{T}$ threshold for jets, reduces this background dramatically. There are also irreducible backgrounds from SM single top events (especially the $s$-channel single top) which are suppressed only at a highly boosted regime in which it is almost impossible for the virtual $\mathrm{W}$ boson to produce a $t \bar{b}$ pair with a high- $p_{T}$ top quark. Using a top tagger, the jet combination is also performed better for final state reconstruction. In the current analysis, there are two $b$-jets in the event. The top tagger tags the $b$-jet inside the fat jet cone as the one from the top quark decay. Using this information, one can reconstruct the top quark invariant mass using jets inside the top tagging cone and reserve the second $b$-jet for the final charged Higgs reconstruction. In a standard search, such combinatorial issues may reduce the signal to background ratio.

There are a large number of articles related to the concept of top tagging from the point of view of the algorithm development and its application to signal selection at LHC. The idea of identifying jet substructure has been discussed in [49-52] while its application in Higgs boson searches (SM and MSSM) can be found in [48, 53, 54].

The general idea of jet substructure is based on finding at least three sub-jets in the hard jet cone satisfying a $p_{T}$ threshold criterion. The starting point is clusterizing input particles into hard jets with a jet algorithm in a large cone, typically, of the size $\Delta R=1.5$. The hard jet is a candidate for the top quark. Therefore it is required to pass a kinematic cut defined as $p_{T}>200 \mathrm{GeV}$ and $\eta<2.5$ where $\eta=-\ln \tan (\theta / 2)$ and $\theta$ is the polar angle with respect to the beam axis. For moderately boosted tops from, e.g., a charged Higgs in the mass range $500 \mathrm{GeV}<m_{H^{ \pm}}<1000 \mathrm{GeV}$, decaying to the top quark, the $p_{T}$ cut can be lowered to $150 \mathrm{GeV}$ [54]. The clustering sequence for the jets is then used for decomposition in a primary step where two parent clusters are identified as candidates for 
the jet pair from $\mathrm{W}$ boson decay and the $b$-jet from the top quark decay. The secondary decomposition then tries to find grandparent clusters by decomposing one of the parent clusters into two, resulting in a final number of at least three clusters. These final clusters are considered as sub-jets from the top quark decay and their invariant mass is required to satisfy the top quark mass window. The invariant mass of the three sub-jets is referred to as the hard jet mass. There are also requirements applied on the jet pairs to check if the invariant mass of one pair satisfies the $\mathrm{W}$ boson mass window as well as a threshold requirement applied on the invariant mass of the jet pair with minimum invariant mass. Details of the cuts, though being algorithm dependent, can be found in [47].

In what was said above, the jet substructure and its application to top tagging was described briefly. However, one may also refer to [55] for a detailed description of the algorithm. The application of fat jets to Higgs searches has been addressed in [56]. The stop reconstruction using top tagging technique has been reported in [57]. Tagging single tops has also been proved to be promising using top tagging algorithm in [58]. There are also reports on how to update the top tagging algorithm for a better fake rate reduction $[59,60]$.

There are several algorithms already available for top tagging. The BDRS [48] is one of the early algorithms based on mass drop criterion in the jet un-clustering. It is originally designed for Higgs decay to bottom quark pair and uses C/A jet algorithm. The YSplitter algorithm [55] is based on the structure of splitting history and is originally developed by ATLAS collaboration for $Z^{\prime}$ searches and is useful for heavy particles with $p_{T}>300 \mathrm{GeV}$. Contrary to the case of YSplitter, the Seatle Tagger [51,52] is based on removing all soft and collinear splittings of QCD jets and studying the remaining massive splittings (the so called "pruning" procedure). The John Hopkins Tagger [47] is one of the first tagging algorithms useful for a two step top decay, starting with a top quark transverse momentum threshold of $1 \mathrm{TeV}$. It is useful for highly boosted top quarks. There is also Thaler-Wang Tagger [49] based on jet energy drop and is useful for top $p_{T}>800 \mathrm{GeV}$.

The HEPTopTagger [57, 59] is a development of BDRS method for multi-step top decays. It is originally desigend for $t \bar{t} H$ searches but can be used for other processes. It starts from a considerably lower top $p_{T}$ and is thus useful for moderately boosted top quarks. While BDRS method relies on the symmetry of the heavy particle decay, the HEPTopTagger does not require a symmetry in the top quark decay. According to the above introduction and comparison of algorithms, the HEPTopTagger is used in this analysis as the top tagging algorithm.

The tagger is said to be useful for top quark $p_{T}$ down to $200 \mathrm{GeV}$ [61]. However, as will be shown, a reasonable tagging efficiency and background suppression is achieved for top quarks with even less $p_{T}$. In the current analysis, two thresholds of 150 and $200 \mathrm{GeV}$ for the top quark $p_{T}$ were tested leading to the conclusion that the cut at $150 \mathrm{GeV}$ is more suitable for this analysis. There are several reasons for that. The cut at $150 \mathrm{GeV}$ avoids the signal statistics reduction especially for low charged Higgs masses for which the cross section is small. Applying $p_{T}>200 \mathrm{GeV}$ reduces both signal and background without improving the signal significance. On the other hand it is not useful for charged Higgs masses as low as $400 \mathrm{GeV}$ for which the top quark $p_{T}$ is below $200 \mathrm{GeV}$ and applying a cut at $200 \mathrm{GeV}$ suppresses almost all the signal. Figure 1 shows the top quark $p_{T}$ in signal events with 


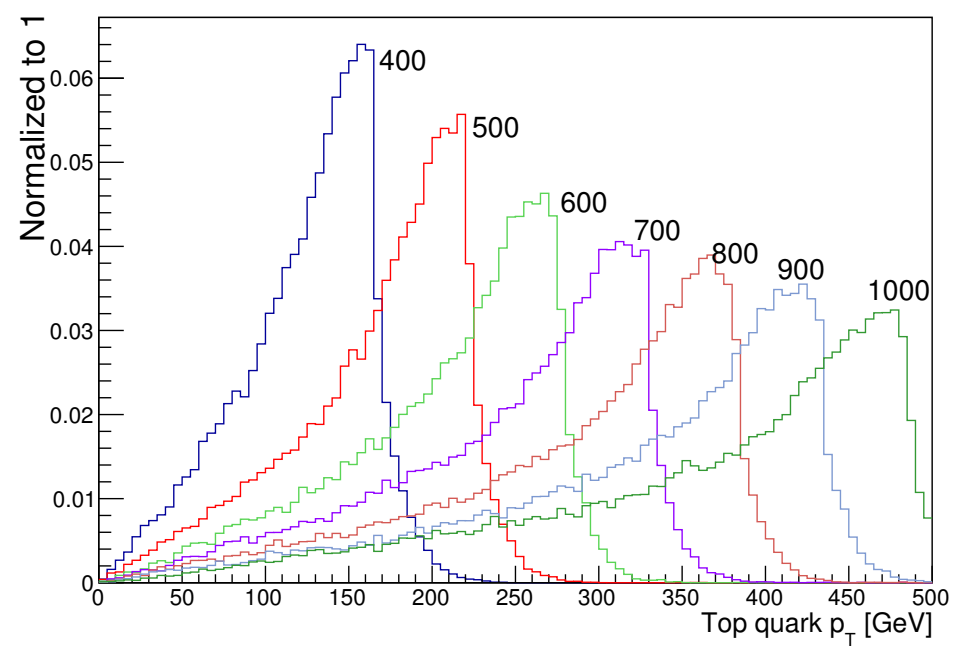

Figure 1. The top quark transverse momentum in signal events for different charged Higgs mass hypotheses. Numbers on histograms correspond to the charged Higgs mass in GeV.

different charged Higgs mass hypotheses. The top tagging has a low efficiency for top quarks produced from $m_{H^{ \pm}}=400 \mathrm{GeV}$, however, it is still able to identify the top quark and its decay constituents, if a threshold of $150 \mathrm{GeV}$ is applied. Therefore, $p_{T}>150 \mathrm{GeV}$ was adopted for the top quark transverse momentum in this analysis.

\section{Signal and background events and their cross sections}

The signal is defined as a process of single charged Higgs production in $s$-channel, followed by the charged Higgs decay to a pair of top and bottom quarks. The theoretical framework is MSSM, $m_{h}$-max scenario [22] with the following parameters: $M_{2}=200 \mathrm{GeV}, M_{\tilde{g}}=$ $800 \mathrm{GeV}, \mu=200 \mathrm{GeV}$ and $M_{S U S Y}=1 \mathrm{TeV}$. The signal cross section depends on the charged Higgs mass as well as $\tan \beta$ as shown in figure 2. These values are obtained using CompHEP 4.5.2 [62, 63] with charged Higgs widths taken from FeynHiggs 2.8.3 [64-66]. Quark masses are taken from Particle Data Group [67]. What is observed here is that the charged Higgs production cross section decreases with increasing its mass, however the reduction can partially be compensated by increasing $\tan \beta$. On the other hand, heavy charged Higgs bosons, may lead to invariant mass distributions lying on the tail of the background distribution thus compensating again partially the small signal cross section in that region. Therefore the two factors of signal cross section and the invariant mass distribution play competing roles.

The main background processes are top quark pair production, single top ( $s$-channel and $t$-channel production) and single gauge boson $\mathrm{W}+$ jets production. The cross section of the top production processes are calculated at NLO using MCFM 6.1 [68-71], while PYTHIA 8.1.53 [72] is used for $\mathrm{W}+$ jets cross section. The used values are listed in table 1. It should be noted that in any event analysis with fully hadronic final state, QCD multijet events may be an important background. As is seen in the next section, they can be 


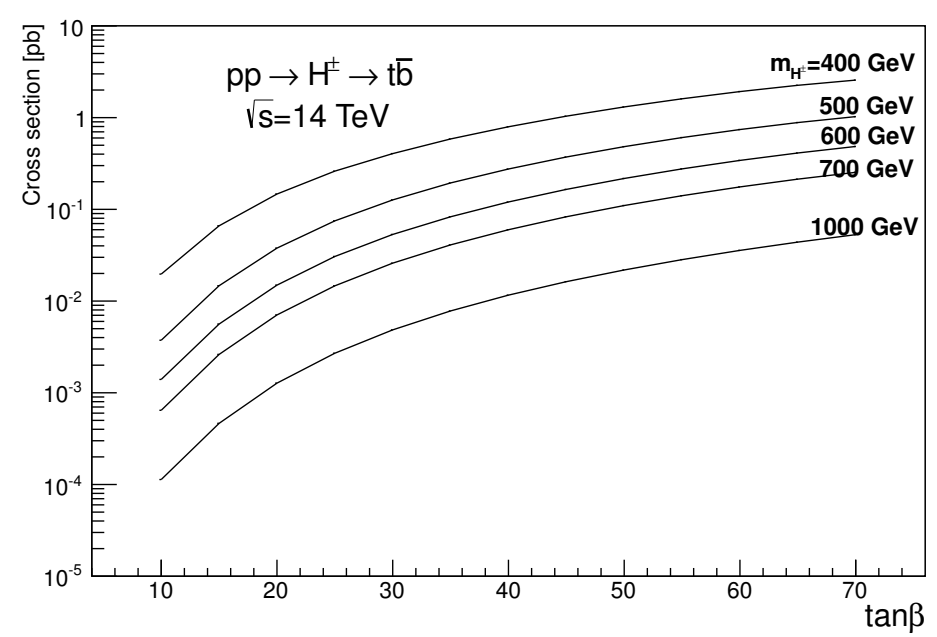

Figure 2. The signal production cross section as a function of $\tan \beta$.

\begin{tabular}{|c|c|c|c|c|}
\hline Process & $t \bar{t}$ & Single top (s-channel) & Single top (t-channel) & W+jets \\
\hline Cross section $[\mathrm{pb}]$ & 834 & 10.5 & 247 & $1.7 \times 10^{5}$ \\
\hline
\end{tabular}

Table 1. Background cross sections.

suppressed by dedicated selection cuts which include the top tagging selection, top and $\mathrm{W}$ mass windows, the $b$-tagging, the jet transverse energy thresholds, etc. At the current analysis, $4 \times 10^{7}$ QCD multi-jet events were analyzed and no event survived.

\section{Event generation and analysis}

Signal events are generated using CompHEP in LHEF format (Les Houches Event File) [73]. The output files are then passed to PYTHIA for further processing including final state showering, multiple interactions, decays, etc. Events are generated with charged Higgs mass hypotheses ranging from $400 \mathrm{GeV}$ to $1 \mathrm{TeV}$ with increments of $100 \mathrm{GeV}$. Background events are all generated using PYTHIA.

Since both signal and background processes are initiated from partons, parton distribution functions (PDF's) are used with a link between LHAPDF 5.8.6 [74] and PYTHIA. In this analysis CTEQ 6.6 [75] is used as the PDF set.

The jet reconstruction is performed using FASTJET 2.4.1 [76, 77] which is a multipurpose jet reconstruction package. A study of different jet reconstruction algoritms in the context of top tagging at LHC [78] has shown that the Cambridge-Aachen algorithm (CA) is better than $k_{T}$ [79] and anti- $k_{T}$ [80] in terms of fake rate suppression and signal from background suppression. Therefore in this analysis, CA algorithm is used as the standard jet reconstruction algorithm.

The strategy of jet reconstruction is similar to what has been adopted in [54] in the sense that a fat jet cone of $\Delta R=1.5$ is used for top jet tagging, while for other jets outside the top decay cone, a standard jet cone with $\Delta R=0.4$ is applied. Therefore the analysis 
uses a "hybrid" jet reconstruction and for each event, the jet reconstruction algorithm is executed twice: once for top tagging, and once for other jets independently.

The top tagging algorithm which is used in this analysis is HepTopTagger [57] with a fat jet cone size of $\Delta R=1.5$ and a jet $p_{T}$ threshold set to $p_{T}>150 \mathrm{GeV}$. Signal events are in fully hadronic final state with a top jet (consisting of two light jets and a $b$-jet in the cone) and a $b$-jet outside the top decay cone. The HEPTopTagger procedure is described as follows.

The last step of $\mathrm{C} / \mathrm{A}$ clustering for reconstruction of fat jets is reversed and the jet $j$ is decomposed into subjets $j_{1}$ and $j_{2}\left(m_{j_{1}} \dot{i} m_{j_{2}}\right)$. If the subjets satisfy mass drop criterion $\min m_{j_{i}}<0.8 m_{j}$, both subjets $j_{1}$ and $j_{2}$ are kept and decomposing continues for both. If the criterion fails, the subjet $j_{2}$ is discarded and decomposing continues only for the subjet $j_{1}$. Throughout this process, each subjet is decomposed if its mass is greater than $30 \mathrm{GeV}$ and it has more than one constituent. Following this method, finally we reach a point, at which the decomposition stops completely and the procedure yields some subjets. In case of finding less than three subjets, the HEPTopTagger fails.

Having subjets in hand, a list of all their possible triplet combinations is created. The constituents of each triplet are now reclustered with $R_{f i l t}=\min \left(0.3, \Delta R_{i j} / 2\right)$, where $i$ and $j$ are the closest subjets. Reclustering yields a number of subjets, of which the five highest transverse momentum ones are kept as filtered subjets, and all others are discarded. This filtering is performed to clean jets from contamination due to the underlying events and pile-up. The filtered mass is defined to be the invariant mass of the five kept filtered subjets. Following this procedure, a set of filtered subjets is obtained for each triplet combination. The set of filtered subjets for which the filtered mass is closest to the top quark mass is kept and all other sets are discarded. The constituents of the kept set of filtered subjets are now again reclustered by exclusive $\mathrm{C} / \mathrm{A}$ algorithm, which causes the jet to have exactly three subjets $j_{1}, j_{2}$ and $j_{3}\left(p_{\mathrm{T}, 1}>p_{\mathrm{T}, 2}>p_{\mathrm{T}, 3}\right)$.

At this point, a mass selection is performed on the final three subjets. The invariant mass $m_{123}$ of the three subjets must be in the top mass window, and the invariant masses $m_{12}, m_{23}$ and $m_{13}$ associated with various pairs of the subjets, must satisfy at least one of the following three conditions:

$$
\begin{aligned}
& 0.2<\arctan \frac{m_{13}}{m_{12}}<1.3 \quad \text { and } R_{\min }<\frac{m_{23}}{m_{123}}<R_{\max } \\
& R_{\min }^{2}\left(1+\left(\frac{m_{13}}{m_{12}}\right)^{2}\right)<1-\left(\frac{m_{23}}{m_{123}}\right)^{2}<R_{\max }^{2}\left(1+\left(\frac{m_{13}}{m_{12}}\right)^{2}\right) \text { and } \frac{m_{23}}{m_{123}}>R_{\mathrm{soft}} \\
& R_{\min }^{2}\left(1+\left(\frac{m_{12}}{m_{13}}\right)^{2}\right)<1-\left(\frac{m_{23}}{m_{123}}\right)^{2}<R_{\max }^{2}\left(1+\left(\frac{m_{12}}{m_{13}}\right)^{2}\right) \quad \text { and } \quad \frac{m_{23}}{m_{123}}>R_{\mathrm{soft}}
\end{aligned}
$$

Where the dimensionless mass windows $R_{\min }=85 \% \times m_{W} / m_{t}$ and $R_{\max }=115 \% \times m_{W} / m_{t}$, and the soft cutoff $R_{\text {soft }}=0.35$ removes QCD events which are not identified as soft radiation by the $\mathrm{C} / \mathrm{A}$ algorithm. The passed subjets are idetified as top quark subjets if they satisfy one another condition. The overall transverse momentum of the three subjets 


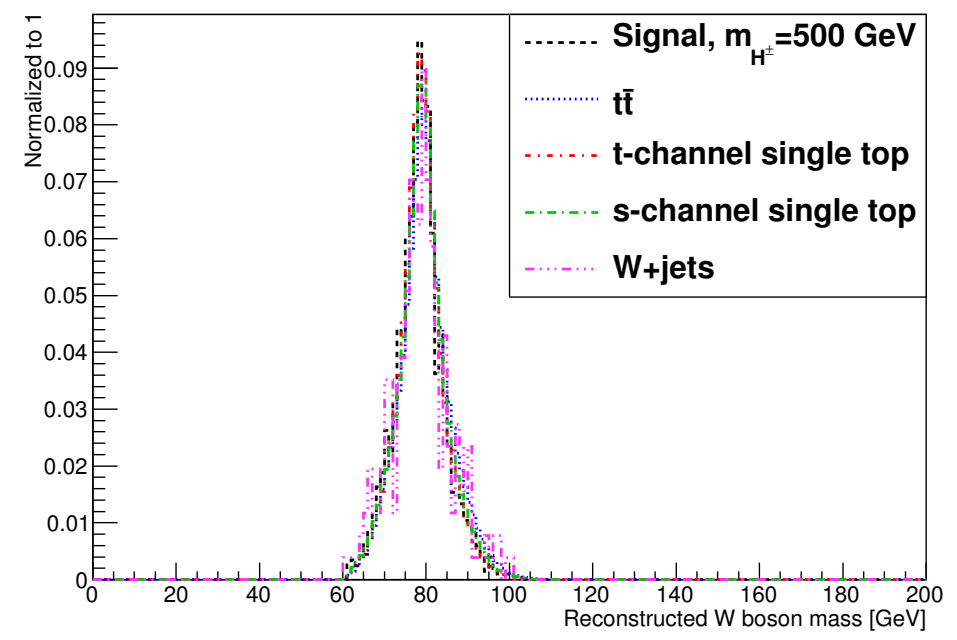

Figure 3. The reconstructed $\mathrm{W}$ boson mass obtained from the top tagging algorithm.

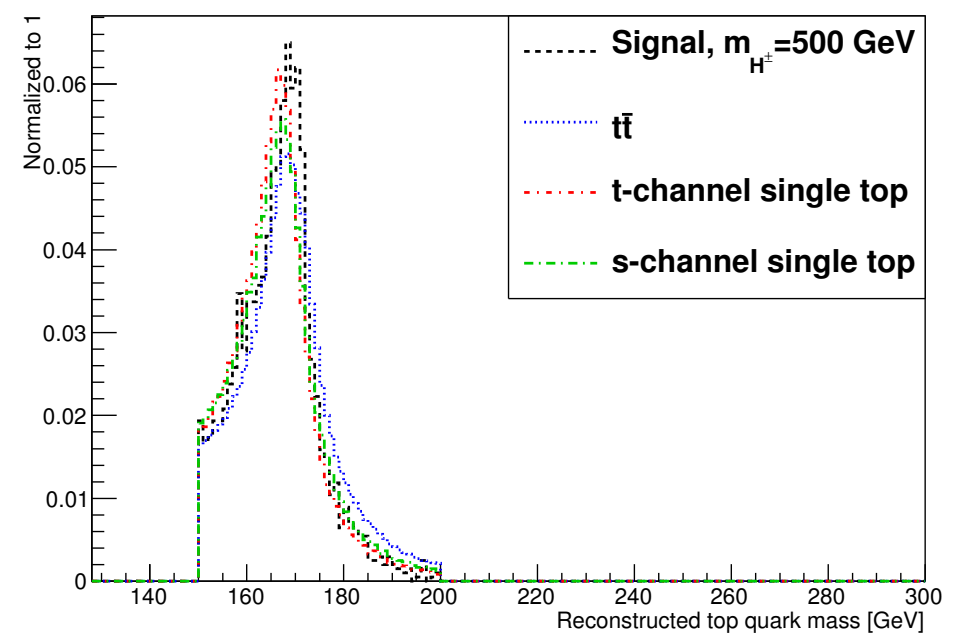

Figure 4. The reconstructed top quark mass obtained from the top tagging algorithm.

must be greater than $150 \mathrm{GeV}$, since the decay jets of the top quark are assumed to be merged in one jet with radius $R=1.5$. Having passed this final check, the top quark identification is completed and the subjets are tagged as top quark subjets.

The final result of the top tagging algorithm is accepted if all three jets satisfy the top mass requirement as in eq. (5.2). Figures 3 and 4 show the distribution of the reconstructed $\mathrm{W}$ boson and top quarks in signal and background events.

$$
150 \mathrm{GeV}<m_{j 1 j 2 b}<190 \mathrm{GeV}
$$

In the next step a search for standard jets is performed and number of jets outside the top decay cone is counted. The kinematic threshold for jets is as in eq. (5.3).

$$
\text { jet } E_{T}>150 \mathrm{GeV}, \quad|\eta|<5
$$




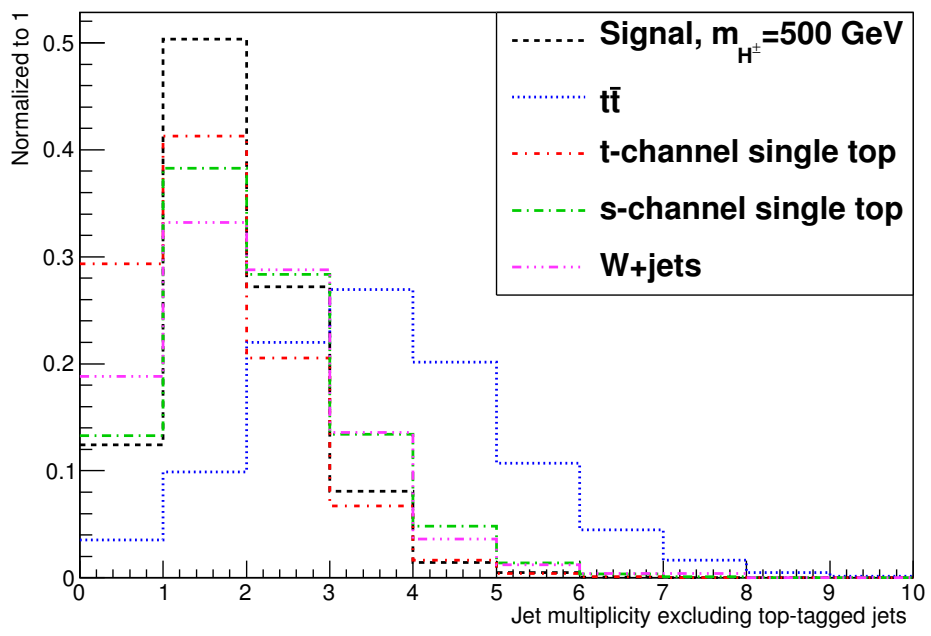

Figure 5. Jet multiplicity excluding jets from the top quark decay.

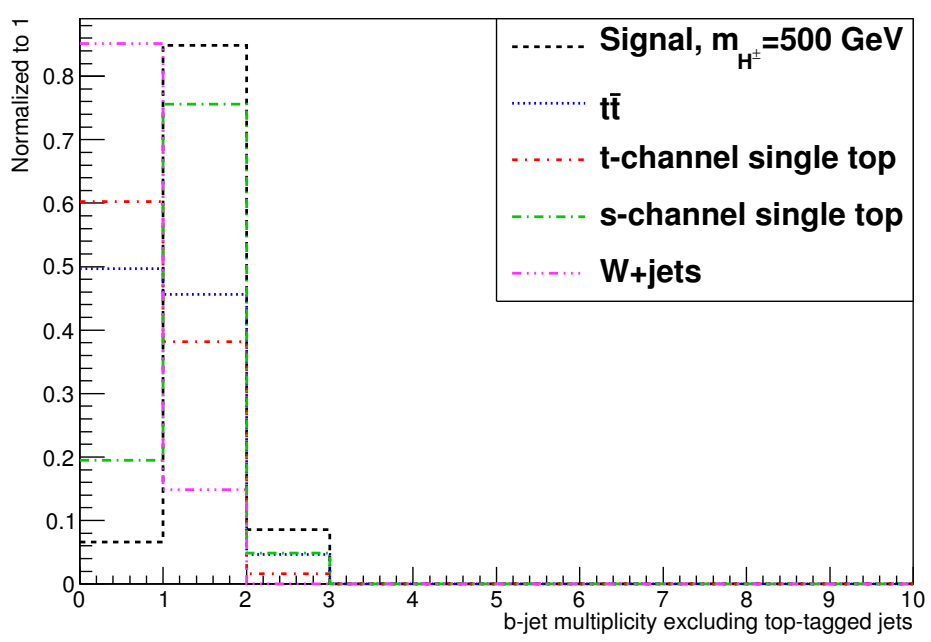

Figure 6. B-jet multiplicity excluding jets from the top quark decay.

Figure 5 shows the total number of jets in signal and background events excluding those which satisfy the top tagging requirements and fall inside the top decay cone. The $b$-tagging is then applied on selected jets in the previous step. For each jet, a search is performed among adjacent $b$ or $c$ quarks using generator level information. A jet is selected as a $b$-jet with $60 \%(10 \%)$ probability if it is near a $b(c)$ quark. The algorithm thus uses the typical $b$-tagging efficiency of LHC experiments [81]. Figure 6 shows number of $b$-jets outside the top decay cone. An event is selected if there is only one $b$-jet outside the top tagging cone.

Another aspect of signal events is that they tend to produce the top and bottom quark pair in opposite directions due to the nature of $s$-channel processes. This feature should appear in azimuthal plane as well. Therefore the angle between the top quark and bottom quark (outside the top decay cone) is calculated and the result is plotted as a distribution 


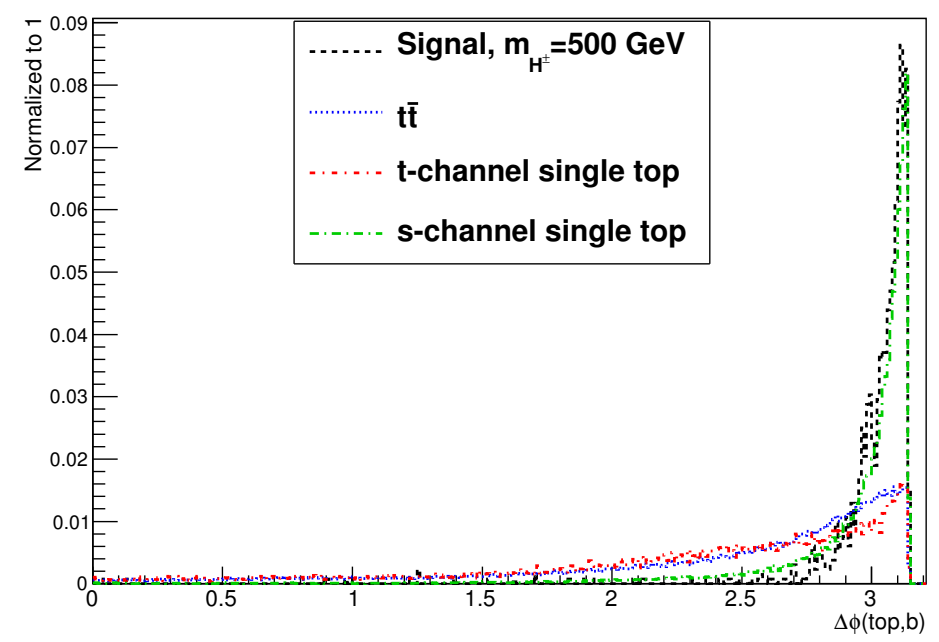

Figure 7. The azimuthal angle difference between the top and bottom quarks.

\begin{tabular}{|c|c|c|c|c|c|c|c|}
\hline$m_{H^{ \pm}}[\mathrm{GeV}]$ & 400 & 500 & 600 & 700 & 800 & 900 & 1000 \\
\hline One tagged top & 0.015 & 0.097 & 0.19 & 0.25 & 0.29 & 0.32 & 0.34 \\
\hline One jet & 0.37 & 0.85 & 0.92 & 0.94 & 0.95 & 0.95 & 0.95 \\
\hline$b$-tagging & 0.9 & 0.97 & 0.98 & 0.97 & 0.97 & 0.98 & 0.98 \\
\hline$\Delta \phi$ (top, bottom $)$ & 0.92 & 0.97 & 0.97 & 0.98 & 0.99 & 0.99 & 0.99 \\
\hline Total eff. & 0.0047 & 0.077 & 0.16 & 0.22 & 0.27 & 0.3 & 0.31 \\
\hline
\end{tabular}

Table 2. Signal selection efficiencies assuming different charged Higgs masses.

\begin{tabular}{|c|c|c|c|c|c|}
\hline Process & $t \bar{t}$ & Single top (s-channel) & Single top (t-channel) & W+jets & QCD \\
\hline One tagged top & 0.13 & 0.023 & 0.017 & $4.9 \mathrm{e}-05$ & $5.8 \mathrm{e}-06$ \\
\hline One jet & 0.47 & 0.73 & 0.62 & 0.62 & 0.61 \\
\hline$b$-tagging & 0.21 & 0.86 & 0.055 & 0.0099 & 0.007 \\
\hline$\Delta \phi($ top, bottom) & 0.73 & 0.91 & 0.82 & 1 & 0 \\
\hline Total eff. & 0.0099 & 0.013 & 0.00047 & $3 \mathrm{e}-07$ & 0 \\
\hline
\end{tabular}

Table 3. Background selection efficiencies.

of $\Delta \phi$ for both signal and background events for comparison as seen in figure 7. According to figure 7 , a selection cut is applied as in eq. (5.4).

$$
\Delta \phi_{(\text {top quark, bottom quark })}>2.8
$$

When all selection cuts are applied, a chain of selection efficiencies is obtained as in table 2 and table 3.In signal events the top and bottom quark come from a charged Higgs boson and their invariant mass should be in principle make the charged Higgs boson mass. However due to jet energy resolution, mis-identification of jets and errors in their energy 


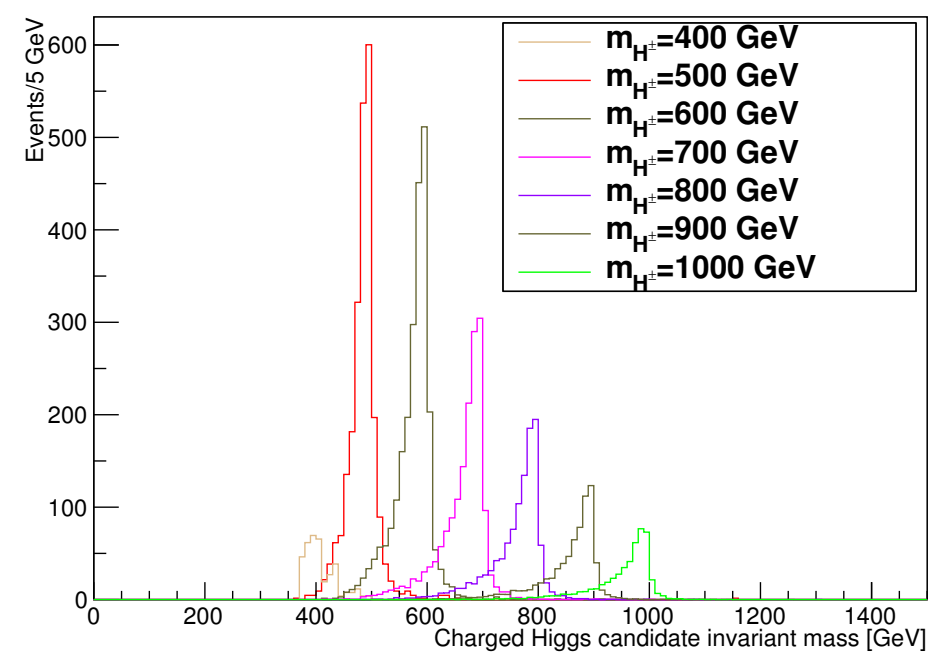

Figure 8. The reconstructed charged Higgs mass in signal events for different charged Higgs mass hypotheses.

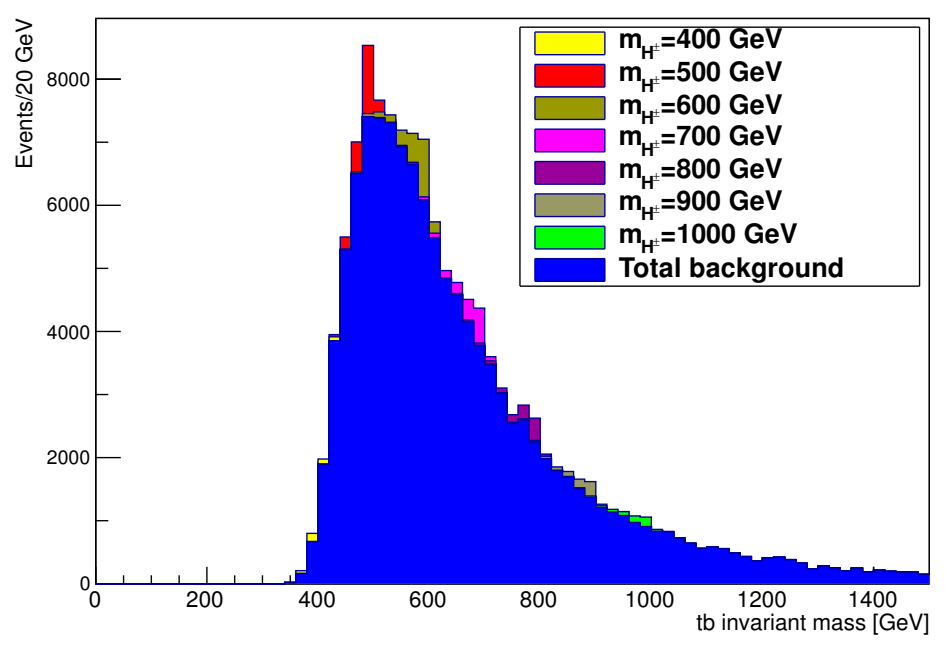

Figure 9. Distribution of signal plus background including different charged Higgs mass peaks independently.

and flight directions, and false jet combination, a distribution of invariant mass with a peak at (almost) the nominal charged Higgs mass is obtained. This distribution is seen in figure 8 where different charged Higgs mass hypotheses are tested in the simulation. The normalization is based on total selection efficiencies (table 2) and cross sections (figure 2) and the integrated luminosity according to $\sigma \times \epsilon \times L$ where $\sigma$ is the signal cross section, $\epsilon$ is the total efficiency and $L$ is the integrated luminosity.

Figure 9 shows signal distributions on top of the total SM background. Due to the large background cross section, signal events appear as small excess of events on top of the background centered at the assumed charged Higgs mass. The natural question is now whether the charged Higgs mass can be extracted from a fit to signal plus background 


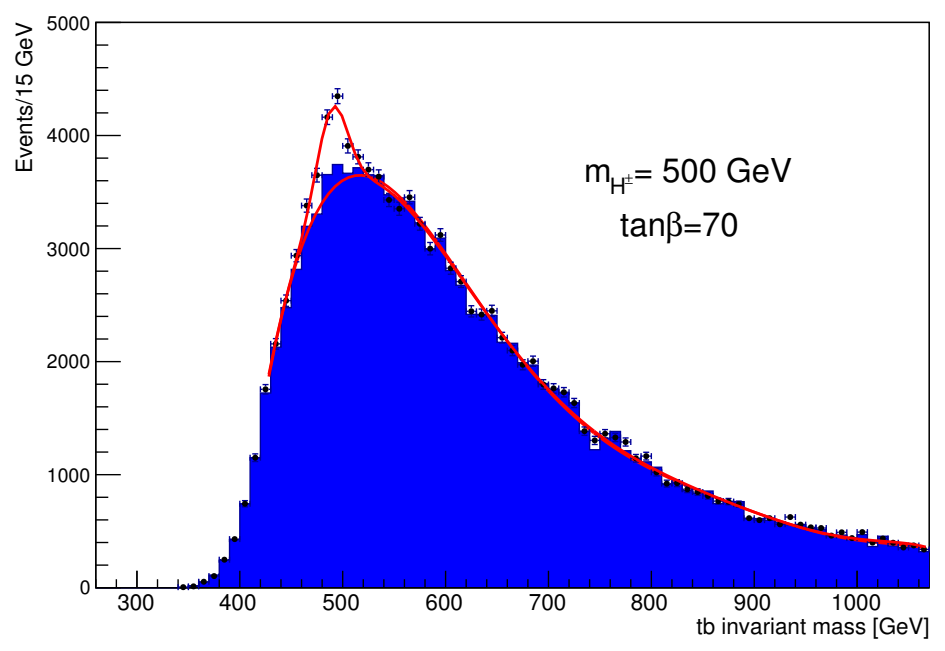

Figure 10. Charged Higgs signal on top of the total background assuming $m_{H^{ \pm}}=500 \mathrm{GeV}$. The pseudo-data are shown with statistical error bars. The background fit and the fit to signal plus background are also shown as solid lines.

distribution. In order to obtain the answer, RooFit package inside ROOT 5.34 [82] is used to simulate toy experiment using probability density distributions taken from signal and background shapes of the charged Higgs candidate invariant mass. In this simulation, for each charged Higgs mass assumption, several toy experiments (LHC's) were performed to check the possibility of the fit to signal and background distributions. Figures 1015 show a typical (selected) experiment demonstrating total background, simulated data with statistical error bars using the PDF's of figures 8 and 9 and the fit to the total distribution. The fit function uses a polinomial and a gaussian function. As seen the signal is well distinguished from the background for charged Higgs masses ranging from 500 to $1000 \mathrm{GeV}$. The $400 \mathrm{GeV}$ charged Higgs could not be well distinguished as it lies on a large and increasing background.

The parameters of the Gaussian part of the fit function can be used to determine the charged Higgs mass. The difference between the reconstructed and generated charged Higgs masses is plotted in figure 16 .

As seen in figure 16, there is an overall negative shift in the mean value of the Gaussian fit with respect to the generated charged Higgs mass. The reason for this should be due to the jet reconstruction algorithm: its parameters (cone size, seed threshold, ...) and the uncertainties arised from them. Since the charged Higgs invariant mass is reconstructed in a fully hadronic environment, the only source of uncertainty should be related to jets. A detailed study of jet four momentum and matching it with the MC truth (i.e. the jet original particle four momentum) could reveal the actual reason for the observed offset. One may also tune the jet algorithm parameters such as the cone size to contain all jet activity inside.

In a real experiment, all sources of uncertainties are taken into account including electronic noise, pile-up, etc. A final correction to the jet four momentum may include 


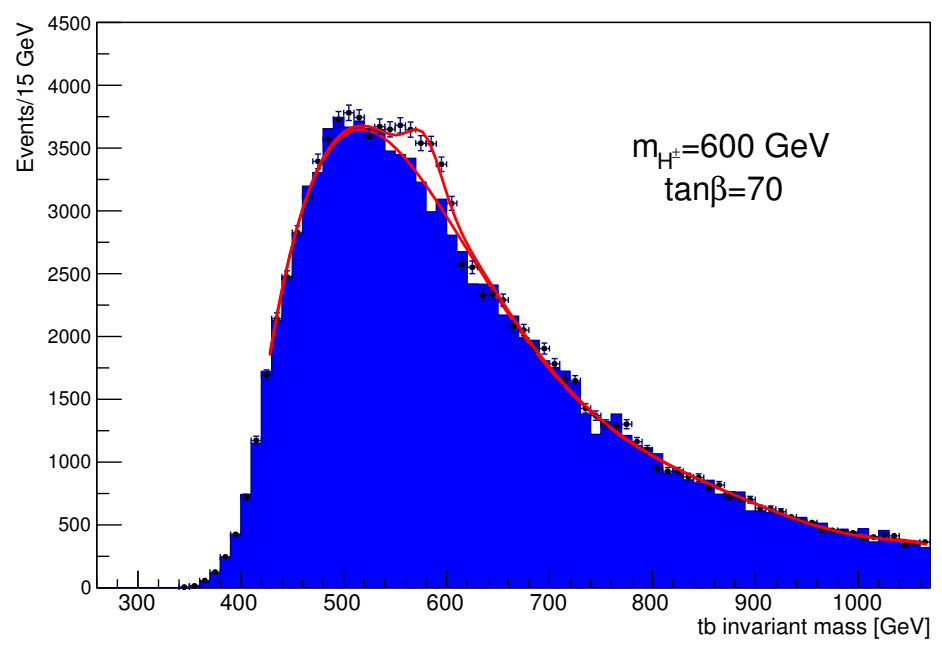

Figure 11. Charged Higgs signal on top of the total background assuming $m_{H^{ \pm}}=600 \mathrm{GeV}$. The pseudo-data are shown with statistical error bars. The background fit and the fit to signal plus background are also shown as solid lines.

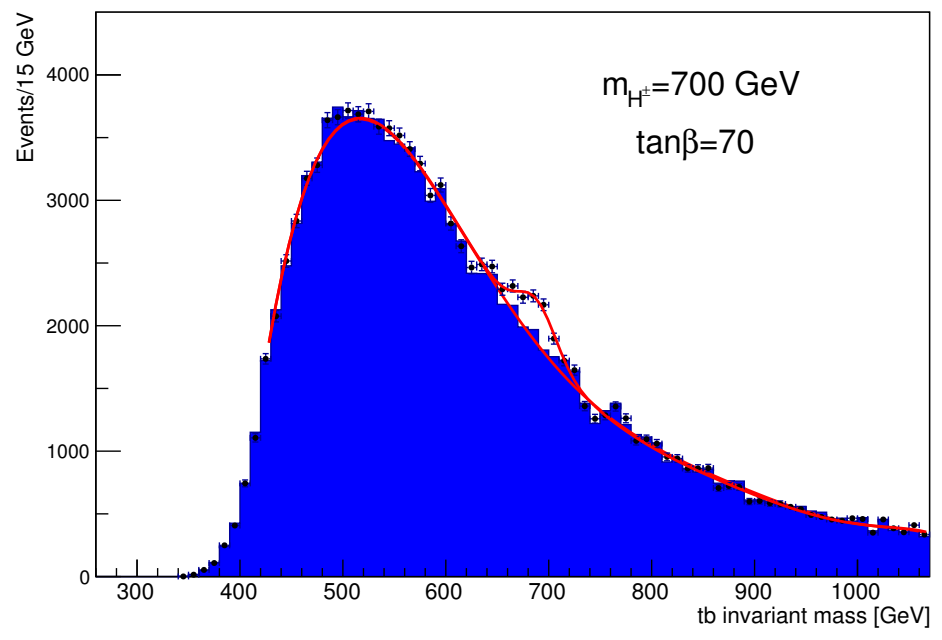

Figure 12. Charged Higgs signal on top of the total background assuming $m_{H^{ \pm}}=700 \mathrm{GeV}$. The pseudo-data are shown with statistical error bars. The background fit and the fit to signal plus background are also shown as solid lines.

several multiplicative factors for off-set effects, Data/MC calibration and jet energy scale uncertainties [83]. Since a study of this type is beyond the scope of this analysis, a simple off-set correction is applied to match reconstructed charged Higgs invariant mass with its true value. In order to do this, a flat function is used to fit to the plot of figure 16 and obtain the average distance of the reconstructed mass from the generated mass. The average value is $-14.8 \mathrm{GeV}$. Therefore all reconstructed masses are increased by $14.8 \mathrm{GeV}$. This correction leads to figure 17 where the difference between the corrected reconstructed masses and generated masses is plotted. 


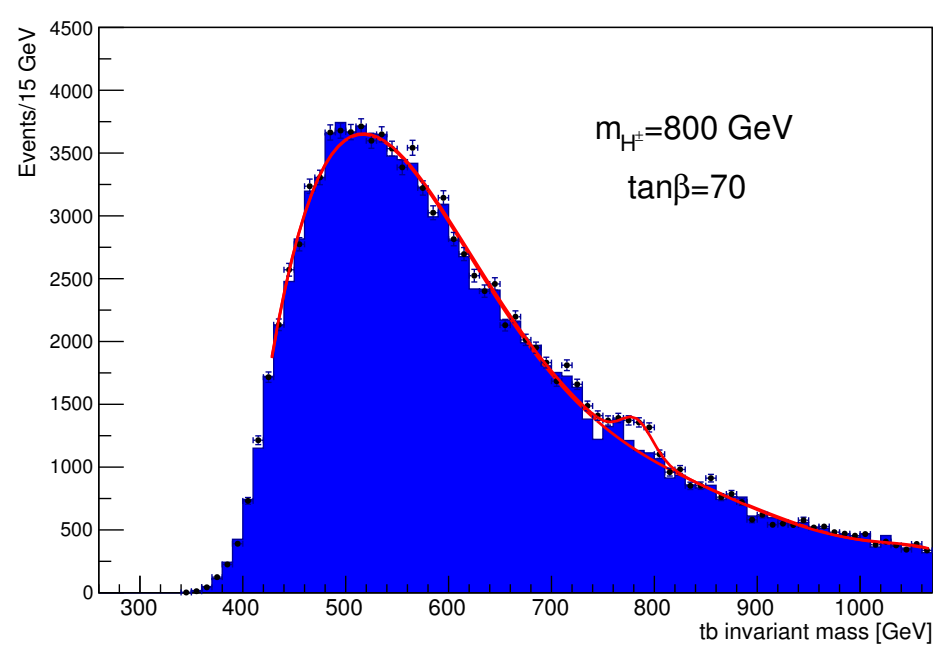

Figure 13. Charged Higgs signal on top of the total background assuming $m_{H^{ \pm}}=800 \mathrm{GeV}$. The pseudo-data are shown with statistical error bars. The background fit and the fit to signal plus background are also shown as solid lines.

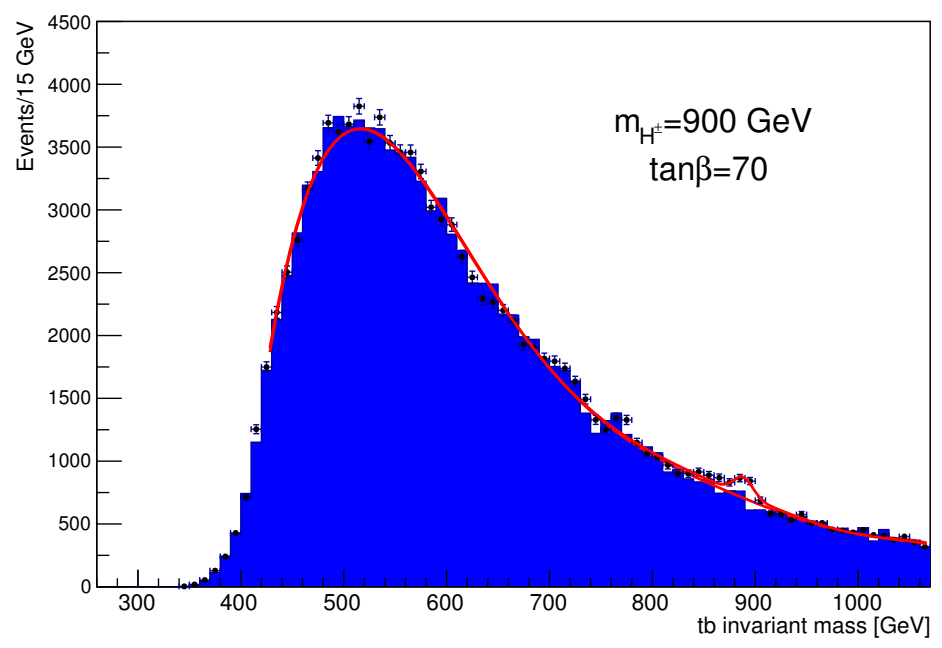

Figure 14. Charged Higgs signal on top of the total background assuming $m_{H^{ \pm}}=900 \mathrm{GeV}$. The pseudo-data are shown with statistical error bars. The background fit and the fit to signal plus background are also shown as solid lines.

The corrected charged Higgs masses are listed in table 4 including fit uncertainties. The charged Higgs mass can thus be measured with few GeV uncertainty. This is however a statistical error. In a real experiment, there are systematic uncertainties which should be taken into account. Since this is a fully hadronic final state analysis, the dominant uncertainty should come from jet energy uncertainty which is expected to be less than $1 \%$ in the central region of detector for jet transverse energies in the range 55 to $500 \mathrm{GeV}$ at the current LHC stage [83]. Other sources of uncertainties include the $b$-tagging uncertainty, the uncertainty from the fit function used in the analysis and the background modeling in 


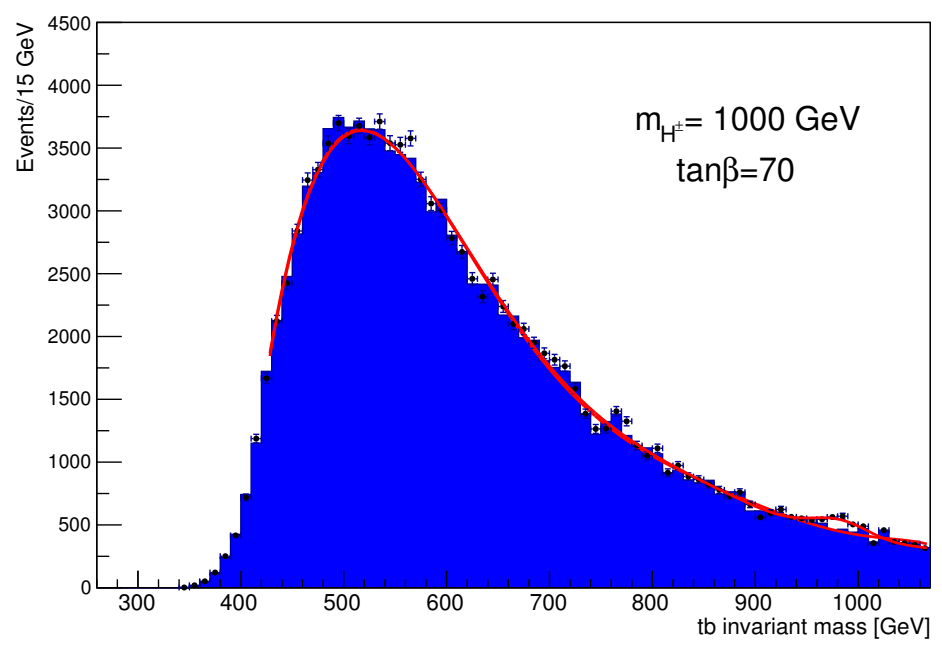

Figure 15. Charged Higgs signal on top of the total background assuming $m_{H^{ \pm}}=1000 \mathrm{GeV}$. The pseudo-data are shown with statistical error bars. The background fit and the fit to signal plus background are also shown as solid lines.

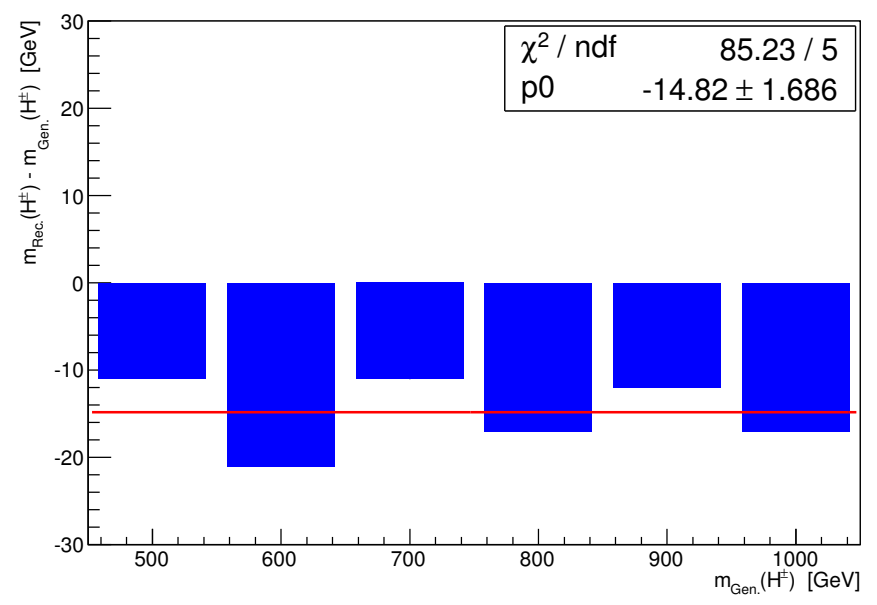

Figure 16. Reconstructed charged Higgs mass minus generated value.

\begin{tabular}{|c|c|c|c|c|c|c|}
\hline Generated mass $[\mathrm{GeV}]$ & 500 & 600 & 700 & 800 & 900 & 1000 \\
\hline Reconstructed mass $[\mathrm{GeV}]$ & $504 \pm 1$ & $594 \pm 3$ & $704 \pm 2$ & $798 \pm 2$ & $903 \pm 3$ & $998 \pm 3$ \\
\hline
\end{tabular}

Table 4. Generated and reconstructed charged Higgs masses. The errors are statistical.

making the total background probability density function $(p d f)$. The latter is an important part of the analysis which relies on a correct understanding of the background distributions. This is well achievable in a real data analysis where distributions of different background samples taken from real data and MC are compared to obtain a reasonable $p d f$ of the total background. 


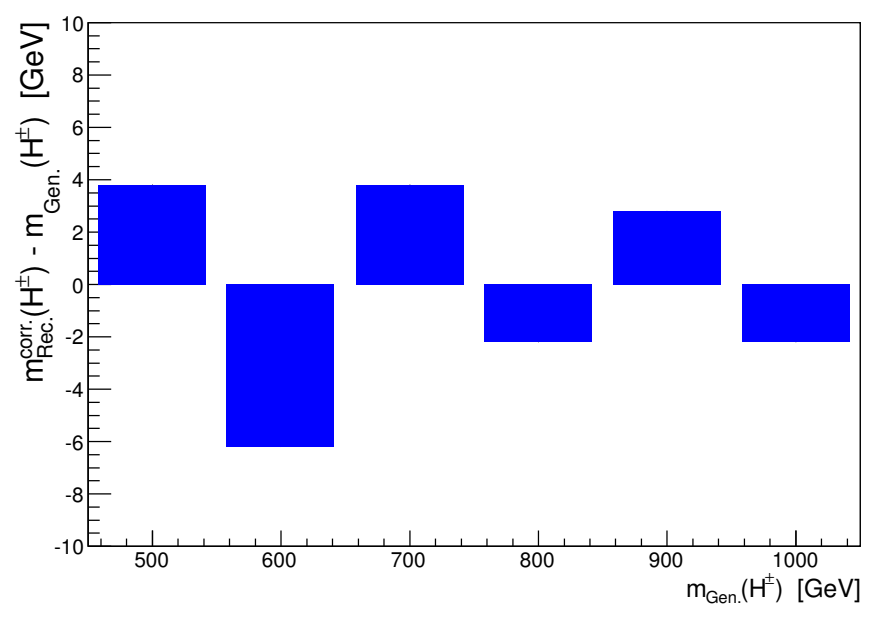

Figure 17. Reconstructed charged Higgs mass minus generated value after correction.

\begin{tabular}{|c|c|c|c|c|c|c|c|}
\hline$m_{H^{ \pm}}[\mathrm{GeV}]$ & 400 & 500 & 600 & 700 & 800 & 900 & 1000 \\
\hline Mass window $[\mathrm{GeV}]$ & $380-400$ & $480-500$ & $570-610$ & $670-700$ & $770-800$ & $870-910$ & $960-1000$ \\
\hline Total eff. & 0.0023 & 0.047 & 0.11 & 0.12 & 0.14 & 0.17 & 0.18 \\
\hline $\mathrm{S}$ & 177 & 1459 & 1660 & 950 & 618 & 440 & 287 \\
\hline $\mathrm{B}$ & 780 & 10702 & 15536 & 7927 & 4839 & 3491 & 2402 \\
\hline$S / B$ & 0.23 & 0.14 & 0.11 & 0.12 & 0.13 & 0.13 & 0.12 \\
\hline$S / \sqrt{B}$ & 6.3 & 14 & 13 & 11 & 8.9 & 7.4 & 5.8 \\
\hline
\end{tabular}

Table 5. Charged Higgs mass window cuts, nutmber of signal and background events after all selection cuts and mass window cut and the final signal to background ratio and signal significance.

\section{Signal significance and phase space contours}

Using the signal plus background distributions of figure 9, a mass window cut is applied to increase the signal significance. The cut window is optimized so as to give the highest significance. Table 5 shows mass window cuts, the total selection efficiency, signal to background ratio and signal significance at $L=30 \mathrm{fb}^{-1}$ and $\tan \beta=70$. In order to obtain the signal significance at other points of $m_{H^{ \pm}}, \tan \beta$ phase space, it is assumed that variation of $\tan \beta$ only changes the cross section due to the change in vertex couplings and does not change the kinematics of events or selection efficiencies. Therefore the signal cross section is calculated at each point of parameter space using CompHep, then TLimit code, which is implemented in ROOT, is used to obtain the signal significance. Results are finally quoted in terms of contours which show the minimum $\tan \beta$ required to exclude a chaged Higgs with a given mass at $95 \%$ confidence level or discover at $5 \sigma$. These contours are shown in figure 18 and 19 where different luminosities have been assumed. The current exclusion contours from LEP and LHC experiments are also included. As seen, LHC exclusion 


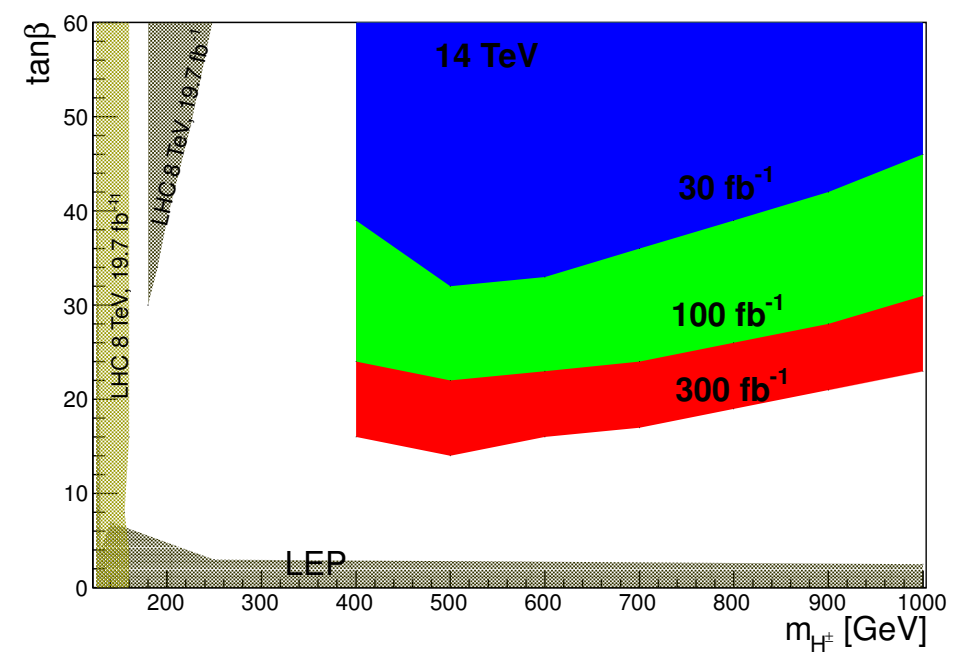

Figure 18. The 95\% C.L. contour as a function of the charged Higgs mass and $\tan \beta$ at different integrated luminosities.

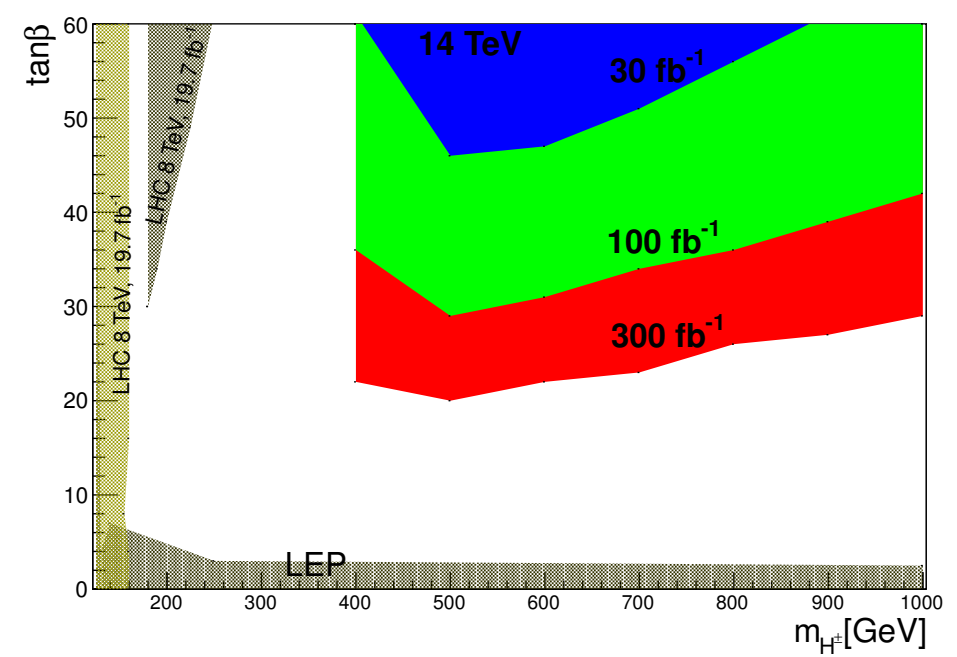

Figure 19. The $5 \sigma$ discovery contour as a function of the charged Higgs mass and $\tan \beta$ at different integrated luminosities.

contour covers a part of high $\tan \beta$ at masses near $200 \mathrm{GeV}$ and is expected to proceed to higher masses and lower $\tan \beta$ values. However, the higher the charged Higgs mass, the lower the cross section. This fact may slow down the progress to heavy charged Higgs area near $0.5 \mathrm{TeV}$. In this region, the analysis presented in this work is expected to help other search analyses.

\section{Conclusions}

The $s$-channel heavy charged Higgs production was studied in the mass range $400<m_{H^{ \pm}}<$ $1000 \mathrm{GeV}$, which is currently outside the reach of LHC. Using the fact that heavy charged 
Higgs with a mass in the range given above, produces boosted top quarks in its decay to $t \bar{b}$ pair, the top tagging technique was used to benefit from the collinearity of jets in the top quark hadronic decay. For other possible jets in the event, ususal jet reconstruction with standard cone size of 0.4 was used independently. Based on kinematic differences of signal and background, selection cuts were applied to select signal events. The increasing top tagging efficiency at higher charged Higgs masses, partially compensates the decrease in signal production cross section. This fact helps observation of charged Higgs at $\mathrm{TeV}$ scale. Results show that using this production process, at a large part of parameter space, an observable signal can be extracted from SM background and the mass measurement is possible in the range 0.5 to $1 \mathrm{TeV}$.

\section{Acknowledgments}

We would like to thank Dr. Mogharrab for his continuous care and maintenance of the computing cluster at Science Department of Shiraz University, without whom and his efforts, this work would not reach its final stage.

Open Access. This article is distributed under the terms of the Creative Commons Attribution License (CC-BY 4.0), which permits any use, distribution and reproduction in any medium, provided the original author(s) and source are credited.

\section{References}

[1] J.F. Gunion, H.E. Haber, G.L. Kane and S. Dawson, The Higgs Hunter's Guide, Front. Phys. 80 (2000) 1.

[2] J.F. Donoghue and L.-F. Li, Properties of charged higgs bosons, Phys. Rev. D 19 (1979) 945.

[3] R.M. Godbole and D.P. Roy, Charged-higgs-boson search via heavy-top-quark decay at fermilab tevatron collider energy, Phys. Rev. D 43 (1991) 3640.

[4] B.K. Bullock, K. Hagiwara and A.D. Martin, Tau polarization as a signal of charged Higgs bosons, Phys. Rev. Lett. 67 (1991) 3055 [INSPIRE].

[5] D.P. Roy, Using $\tau$ polarization for the charged Higgs search at hadron colliders, Phys. Lett. B 277 (1992) 183 [INSPIRE].

[6] S. Raychaudhuri and D.P. Roy, Charged Higgs boson search at the Tevatron upgrade using $\tau$ polarization, Phys. Rev. D 52 (1995) 1556 [hep-ph/9503251] [INSPIRE].

[7] D. Roy, The hadronic tau decay signature of a heavy charged higgs boson at LHC, Phys. Lett. B 459 (1999) 607.

[8] S. Raychaudhuri and D.P. Roy, Sharpening up the charged Higgs boson signature using $\tau$ polarization at LHC, Phys. Rev. D 53 (1996) 4902 [hep-ph/9507388] [INSPIRE].

[9] J.L. Diaz-Cruz and O.A. Sampayo, Contribution of gluon fusion to the production of charged Higgs at hadron colliders, Phys. Rev. D 50 (1994) 6820 [INSPIRE].

[10] J. Gunion, Detecting the tb decays of a charged higgs boson at a hadron supercollider, Phys. Lett. B 322 (1994) 125. 
[11] V.D. Barger, R.J.N. Phillips and D.P. Roy, Heavy charged Higgs signals at the LHC, Phys. Lett. B 324 (1994) 236 [hep-ph/9311372] [INSPIRE].

[12] E. Ma, D.P. Roy and J. Wudka, Enhanced three-body decay of the charged Higgs boson, Phys. Rev. Lett. 80 (1998) 1162 [hep-ph/9710447] [INSPIRE].

[13] A. Krause, T. Plehn, M. Spira and P.M. Zerwas, Production of charged Higgs boson pairs in gluon-gluon collisions, Nucl. Phys. B 519 (1998) 85 [hep-ph/9707430] [INSPIRE].

[14] S. Moretti and D.P. Roy, Detecting heavy charged Higgs bosons at the LHC with triple $b$ tagging, Phys. Lett. B 470 (1999) 209 [hep-ph/9909435] [INSPIRE].

[15] T. Plehn, Charged Higgs boson production in bottom gluon fusion, Phys. Rev. D 67 (2003) 014018 [hep-ph/0206121] [INSPIRE].

[16] N. Kidonakis, Charged Higgs production via bg $\rightarrow$ th $^{-}$at the LHC, JHEP 05 (2005) 011 [hep-ph/0412422] [INSPIRE].

[17] E.L. Berger, T. Han, J. Jiang and T. Plehn, Associated production of a top quark and a charged higgs boson, Phys. Rev. D 71 (2005) 115012.

[18] F. Mahmoudi and O. Stal, Flavor constraints on the two-Higgs-doublet model with general Yukawa couplings, Phys. Rev. D 81 (2010) 035016 [arXiv:0907.1791] [INSPIRE].

[19] ALEPH collaboration, R. Barate et al., Search for charged Higgs bosons in $e^{+} e^{-}$collisions at energies up to $\sqrt{s}=189 \mathrm{GeV}$, Phys. Lett. B 487 (2000) 253 [hep-ex/0008005] [INSPIRE].

[20] L3 collaboration, M. Acciarri et al., Search for charged Higgs bosons in $e^{+} e^{-}$collisions at center center-of-mass energies up to 202-GeV, Phys. Lett. B 496 (2000) 34 [hep-ex/0009010] [INSPIRE].

[21] OPAL, DELPHI, L3, AlEPH, LEP Higgs Working Group for Higgs boson SEARCHES collaborations, Search for charged Higgs bosons: preliminary combined LEP data collected at energies up to $209 \mathrm{GeV}$, in Lepton and photon interactions at high energies, proceedings of the 20th International Symposium, LP 2001, Rome, Italy, 23-28 July 2001 [hep-ex/0107031].

[22] DELPHI, OPAL, ALEPH, LEP Higgs Working Group, L3 collaborations, Searches for the neutral Higgs bosons of the MSSM: preliminary combined results using LEP data collected at energies up to $209 \mathrm{GeV}$, in Lepton and photon interactions at high energies, proceedings of the 20th International Symposium, LP 2001, Rome, Italy, 23-28 July 2001 [hep-ex/0107030].

[23] D0 collaboration, B. Abbott et al., Search for charged Higgs bosons in decays of top quark pairs, Phys. Rev. Lett. 82 (1999) 4975 [hep-ex/9902028] [INSPIRE].

[24] D0 collaboration, V.M. Abazov et al., Search for charged Higgs bosons in decays of top quarks, Phys. Rev. D 80 (2009) 051107 [arXiv:0906.5326] [INSPIRE].

[25] D0 collaboration, V.M. Abazov et al., Search for Charged Higgs Bosons in Top Quark Decays, Phys. Lett. B 682 (2009) 278 [arXiv:0908.1811] [INSPIRE].

[26] D0 collaboration, V.M. Abazov et al., Combination of $t \bar{t}$ cross section measurements and constraints on the mass of the top quark and its decays into charged Higgs bosons, Phys. Rev. D 80 (2009) 071102 [arXiv:0903.5525] [INSPIRE]. 
[27] CDF collaboration, A. Abulencia et al., Search for charged Higgs bosons from top quark decays in $p \bar{p}$ collisions at $\sqrt{s}=1.96$ TeV., Phys. Rev. Lett. 96 (2006) 042003 [hep-ex/0510065] [INSPIRE].

[28] CDF collaboration, G. Yu, Search for charged Higgs in top quark decays at CDF, AIP Conf. Proc. 1078 (2008) 198 [INSPIRE].

[29] CDF collaboration, T. Aaltonen et al., Search for charged Higgs bosons in decays of top quarks in $p \bar{p}$ collisions at $\sqrt{s}=1.96$ TeV, Phys. Rev. Lett. 103 (2009) 101803 [arXiv:0907.1269] [INSPIRE].

[30] M. Baarmand, M. Hashemi and A. Nikitenko, Light charged Higgs discovery potential of $C M S$ in the $H^{ \pm} \rightarrow \tau \nu_{\tau}$ decay with single lepton trigger, J. Phys. G 32 (2006) N21 [INSPIRE].

[31] C. Biscarat and M. Dosil, Charged Higgs search in top quark decays with the ATLAS detector, ATL-PHYS-2003-038 (2003).

[32] CMS collaboration, Search for a light charged Higgs boson in top quark decays in pp collisions at $\sqrt{s}=7$ TeV, JHEP 07 (2012) 143 [arXiv:1205.5736] [INSPIRE].

[33] CMS Collaboration, Updated search for a light charged Higgs boson in top quark decays in $p p$ collisions at $\sqrt{s}=7$ TeV, CMS-PAS-HIG-12-052.

[34] ATLAS collaboration, Search for charged Higgs bosons decaying via $H^{+} \rightarrow \tau \nu$ in top quark pair events using pp collision data at $\sqrt{s}=7$ TeV with the ATLAS detector, JHEP 06 (2012) 039 [arXiv: 1204.2760] [INSPIRE].

[35] ATLAS collaboration, Search for a light charged Higgs boson in the decay channel $H^{+} \rightarrow c \bar{s}$ in $t \bar{t}$ events using pp collisions at $\sqrt{s}=7$ TeV with the ATLAS detector, Eur. Phys. J. C 73 (2013) 2465 [arXiv: 1302.3694] [INSPIRE].

[36] S. Lowette, J. D'Hondt, and P. Vanlaer, Charged MSSM Higgs Boson Observability in the $H^{+-} \rightarrow t b$ Decay, CERN-CMS-NOTE-2006-109.

[37] R. Kinnunen, Search for the Heavy Charged MSSM Higgs bosons with the $H^{ \pm} \rightarrow \tau^{ \pm} \nu_{\tau}$ Decay Mode in Fully Hadronic Final State, CMS-NOTE-2006-100.

[38] K.A. Assamagan, Y. Coadou and A. Deandrea, ATLAS discovery potential for a heavy charged Higgs boson, Eur. Phys. J. direct C 4 (2002) 9 [hep-ph/0203121] [InSPIRE].

[39] K.A. Assamagan and N. Gollub, The ATLAS discovery potential for a heavy charged Higgs boson in $g g \rightarrow t b H+-$ with $H^{ \pm} \rightarrow t b$, Eur. Phys. J. C 39S2 (2005) 25 [hep-ph/0406013] [INSPIRE].

[40] CMS Collaboration, Search for charged Higgs bosons with the H+ to tau nu decay channel in the fully hadronic final state at sqrt $s=8 \mathrm{TeV}$, CMS-PAS-HIG-14-020.

[41] ATLAS collaboration, Search for charged Higgs bosons decaying via $H^{ \pm} \rightarrow \tau^{ \pm} \nu$ in fully hadronic final states using pp collision data at $\sqrt{s}=8$ TeV with the ATLAS detector, JHEP 03 (2015) 088 [arXiv: 1412.6663] [INSPIRE].

[42] ATLAS collaboration, Search for charged Higgs bosons through the violation of lepton universality in $t \bar{t}$ events using pp collision data at $\sqrt{s}=7$ TeV with the ATLAS experiment, JHEP 03 (2013) 076 [arXiv: 1212.3572] [INSPIRE].

[43] M. Hashemi, Single Top Events as a Source of Light Charged Higgs in the Fully Hadronic Final State at LHC, JHEP 05 (2013) 112 [arXiv: 1305.2096] [INSPIRE]. 
[44] M. Hashemi, Observability of Heavy Charged Higgs through s-channel Single Top Events at LHC, JHEP 11 (2013) 005 [arXiv:1310.5209] [INSPIRE].

[45] M. Hashemi, S.M. Zebarjad and H. Bakhshalizadeh, Off-diagonal Yukawa Couplings in the s-channel Charged Higgs Production at LHC, Phys. Lett. B 741 (2015) 145 [arXiv: 1407.3436] [INSPIRE].

[46] I.I.Y. Bigi, Y.L. Dokshitzer, V.A. Khoze, J.H. Kuhn and P.M. Zerwas, Production and Decay Properties of Ultraheavy Quarks, Phys. Lett. B 181 (1986) 157 [INSPIRE].

[47] D.E. Kaplan, K. Rehermann, M.D. Schwartz and B. Tweedie, Top Tagging: A Method for Identifying Boosted Hadronically Decaying Top Quarks, Phys. Rev. Lett. 101 (2008) 142001 [arXiv: 0806.0848] [INSPIRE].

[48] J.M. Butterworth, A.R. Davison, M. Rubin and G.P. Salam, Jet substructure as a new Higgs search channel at the LHC, Phys. Rev. Lett. 100 (2008) 242001 [arXiv:0802.2470] [INSPIRE].

[49] J. Thaler and L.-T. Wang, Strategies to Identify Boosted Tops, JHEP 07 (2008) 092 [arXiv: 0806.0023] [INSPIRE].

[50] L.G. Almeida, S.J. Lee, G. Perez, I. Sung and J. Virzi, Top Jets at the LHC, Phys. Rev. D 79 (2009) 074012 [arXiv:0810.0934] [INSPIRE].

[51] S.D. Ellis, C.K. Vermilion and J.R. Walsh, Techniques for improved heavy particle searches with jet substructure, Phys. Rev. D 80 (2009) 051501 [arXiv:0903.5081] [INSPIRE].

[52] S.D. Ellis, C.K. Vermilion and J.R. Walsh, Recombination Algorithms and Jet Substructure: Pruning as a Tool for Heavy Particle Searches, Phys. Rev. D 81 (2010) 094023 [arXiv:0912.0033] [INSPIRE].

[53] G.D. Kribs, A. Martin, T.S. Roy and M. Spannowsky, Discovering the Higgs Boson in New Physics Events using Jet Substructure, Phys. Rev. D 81 (2010) 111501 [arXiv:0912.4731] [INSPIRE].

[54] S. Yang and Q.-S. Yan, Searching for Heavy Charged Higgs Boson with Jet Substructure at the LHC, JHEP 02 (2012) 074 [arXiv:1111.4530] [INSPIRE].

[55] J.M. Butterworth, B.E. Cox and J.R. Forshaw, $W W$ scattering at the CERN LHC, Phys. Rev. D 65 (2002) 096014 [hep-ph/0201098] [INSPIRE].

[56] T. Plehn, G.P. Salam and M. Spannowsky, Fat Jets for a Light Higgs, Phys. Rev. Lett. 104 (2010) 111801 [arXiv:0910.5472] [INSPIRE].

[57] T. Plehn, M. Spannowsky, M. Takeuchi and D. Zerwas, Stop Reconstruction with Tagged Tops, JHEP 10 (2010) 078 [arXiv:1006.2833] [INSPIRE].

[58] F. Kling, T. Plehn and M. Takeuchi, Tagging single Tops, Phys. Rev. D 86 (2012) 094029 [arXiv: 1207.4787] [INSPIRE].

[59] T. Plehn, M. Spannowsky and M. Takeuchi, How to Improve Top Tagging, Phys. Rev. D 85 (2012) 034029 [arXiv:1111.5034] [rnSPIRE].

[60] G. Kasieczka, T. Plehn, T. Schell, T. Strebler and G.P. Salam, Resonance Searches with an Updated Top Tagger, JHEP 06 (2015) 203 [arXiv:1503.05921] [INSPIRE].

[61] T. Plehn and M. Spannowsky, Top Tagging, J. Phys. G 39 (2012) 083001 [arXiv:1112.4441] [INSPIRE]. 
[62] CompHEP collaboration, E. Boos, V. Bunichev, M. Dubinin, L. Dudko, V. Ilyin, A. Kryukov et al., CompHEP 4.4: Automatic computations from Lagrangians to events, Nucl. Instrum. Meth. A 534 (2004) 250 [hep-ph/0403113] [INSPIRE].

[63] A. Pukhov, E. Boos, M. Dubinin, V. Edneral, V. Ilyin, D. Kovalenko et al., CompHEP: A Package for evaluation of Feynman diagrams and integration over multiparticle phase space, hep-ph/9908288 [INSPIRE].

[64] S. Heinemeyer, W. Hollik and G. Weiglein, FeynHiggs: A Program for the calculation of the masses of the neutral CP even Higgs bosons in the MSSM, Comput. Phys. Commun. 124 (2000) 76 [hep-ph/9812320] [INSPIRE].

[65] T. Hahn, S. Heinemeyer, W. Hollik, H. Rzehak and G. Weiglein, FeynHiggs: A program for the calculation of MSSM Higgs-boson observables - Version 2.6.5, Comput. Phys. Commun. 180 (2009) 1426 [INSPIRE].

[66] T. Hahn, S. Heinemeyer, W. Hollik, H. Rzehak and G. Weiglein, FeynHiggs 2.7, Nucl. Phys. Proc. Suppl. 205-206 (2010) 152 [arXiv: 1007.0956] [INSPIRE].

[67] Particle Data Group collaboration, K.A. Olive et al., Review of Particle Physics, Chin. Phys. C 38 (2014) 090001 [INSPIRE].

[68] J.M. Campbell, R.K. Ellis and F. Tramontano, Single top production and decay at next-to-leading order, Phys. Rev. D 70 (2004) 094012 [hep-ph/0408158] [INSPIRE].

[69] J.M. Campbell and F. Tramontano, Next-to-leading order corrections to Wt production and decay, Nucl. Phys. B 726 (2005) 109 [hep-ph/0506289] [InSPIRE].

[70] J.M. Campbell, R. Frederix, F. Maltoni and F. Tramontano, Next-to-Leading-Order Predictions for t-Channel Single-Top Production at Hadron Colliders, Phys. Rev. Lett. 102 (2009) 182003 [arXiv:0903.0005] [INSPIRE].

[71] J.M. Campbell and R.K. Ellis, Top-quark processes at NLO in production and decay, J. Phys. G 42 (2015) 015005 [arXiv: 1204.1513] [INSPIRE].

[72] T. Sjöstrand, S. Mrenna and P.Z. Skands, A Brief Introduction to PYTHIA 8.1, Comput. Phys. Commun. 178 (2008) 852 [arXiv:0710.3820] [INSPIRE].

[73] J. Alwall et al., A Standard format for Les Houches event files, Comput. Phys. Commun. 176 (2007) 300 [hep-ph/0609017] [INSPIRE].

[74] A. Buckley, J. Ferrando, S. Lloyd, K. Nordström, B. Page, M. Rüfenacht et al., LHAPDF6: parton density access in the LHC precision era, Eur. Phys. J. C 75 (2015) 132 [arXiv:1412.7420] [INSPIRE].

[75] P.M. Nadolsky, H.-L. Lai, Q.-H. Cao, J. Huston, J. Pumplin, D. Stump et al., Implications of CTEQ global analysis for collider observables, Phys. Rev. D 78 (2008) 013004 [arXiv: 0802.0007] [INSPIRE].

[76] M. Cacciari, FastJet: a code for fast $k_{t}$ clustering, and more, in Deep inelastic scattering, proceedings of the 14th International Workshop, DIS 2006, Tsukuba, Japan, 20-24 April 2006, pg. 487-490 [hep-ph/0607071].

[77] M. Cacciari, G.P. Salam and G. Soyez, FastJet User Manual, Eur. Phys. J. C 72 (2012) 1896 [arXiv: 1111.6097] [INSPIRE].

[78] CMS Collaboration, A Cambridge-Aachen (C-A) based Jet Algorithm for boosted top-jet tagging, CMS-PAS-JME-09-001. 
[79] S. Catani, Y.L. Dokshitzer, M.H. Seymour and B.R. Webber, Longitudinally invariant $K_{t}$ clustering algorithms for hadron hadron collisions, Nucl. Phys. B 406 (1993) 187 [INSPIRE].

[80] M. Cacciari, G.P. Salam and G. Soyez, The Anti-k(t) jet clustering algorithm, JHEP 04 (2008) 063 [arXiv: 0802.1189] [INSPIRE].

[81] CMS collaboration, Identification of b-quark jets with the CMS experiment, 2013 JINST 8 P04013 [arXiv: 1211.4462] [INSPIRE].

[82] R. Brun and F. Rademakers, ROOT: An object oriented data analysis framework, Nucl. Instrum. Meth. A 389 (1997) 81 [INSPIRE].

[83] ATLAS collaboration, Jet energy measurement and its systematic uncertainty in proton-proton collisions at $\sqrt{s}=7 \mathrm{TeV}$ with the ATLAS detector, Eur. Phys. J. C 75 (2015) 17 [arXiv: 1406.0076] [INSPIRE]. 